\title{
Goniometric characteristics of optical fibres for temperature measurement in diesel engine exhaust filters
}

\author{
Brian J Boothe ${ }^{1}$, Albert J Shih ${ }^{2,3}$, Jian Kong ${ }^{1}$ and \\ William L Roberts ${ }^{1}$ \\ ${ }^{1}$ Department of Mechanical and Aerospace Engineering, North Carolina State University, \\ Raleigh, NC 27695, USA \\ ${ }^{2}$ Department of Mechanical Engineering, University of Michigan, Ann Arbor, MI 48109, USA \\ E-mail: shiha@umich.edu
}

Received 29 November 2002, in final form 14 February 2003, accepted for publication 25 February 2003

Published 21 March 2003

Online at stacks.iop.org/MST/14/563

\begin{abstract}
The accurate in situ, non-contact measurement of the temperature distribution within diesel after-treatment filters requires the employment of optical fibres with special tip geometry. The goniometric characteristics of optical fibres with flat, $45^{\circ}$ angled and bent and polished tips are studied such that the specific radiation acceptance region can be determined. One $2 \mathrm{~mm}$ diameter fused silica and two $0.425 \mathrm{~mm}$ diameter sapphire optical fibres are examined. Detailed discussion of the relative intensity profiles observed for these fibres is presented. Of the three fibres evaluated, the $45^{\circ}$ angled tip geometry provides the most precise response for measuring radiation emitted from the internal filter walls. Exploiting the characteristics of total internal reflection, the $45^{\circ}$ angled tip fibre accepts the maximum quantity of incident radiation at an angle perpendicular to the optical axis.
\end{abstract}

Keywords: goniometric characteristics, optical fibre, angled tip, effective numerical aperture

\section{Introduction}

The application of optical fibres for infrared temperature measurement has further evolved with the development of diesel engine exhaust after-treatment filter technologies. Diesel exhaust after-treatment filters are designed to capture and periodically remove environmentally harmful $\mathrm{NO}_{x}$ and particulates [1]. These filters are typically made of porous ceramic material with deep cavities to increase the specific surface area. The temperature at the filter surfaces, due to microwave, post-combustion burning or various other means of heating, controls the chemical kinetics of the storage and regeneration processes. A uniform temperature distribution is critical for controlling the chemical reactions as well as avoiding thermal stress fatigue and overheating of the filter. Accurate temperature measurements of a

3 Author to whom any correspondence should be addressed.
\end{abstract}

small and specific region of the filter wall provide important information to chemical engineers for catalytic development and to mechanical engineers for design of the filter heating process [2].

Attaching traditional thermocouples to the porous ceramic wall at locations deep within the diesel exhaust after-treatment filter cavity has proven difficult due to space constraints. The filter also operates in a harsh environment; high temperature airflow from the engine combustion process as well as thermal cycling as a consequence of periodical regeneration. Furthermore, the ceramic filter responds rapidly to microwave heating, precluding the use of traditional thermocouples due to their inherently metallic composition. As an alternative to using a thermocouple, an optical fibre is employed to transmit emitted surface radiation to an infrared detector for temperature measurement, as illustrated in figure 1 . With typical operating temperatures ranging from 400 to $800{ }^{\circ} \mathrm{C}$, 


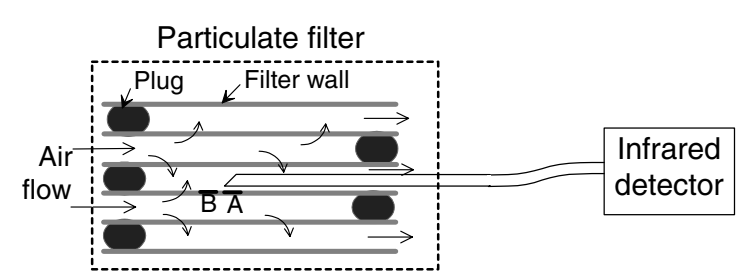

Figure 1. Application of an angled tip optical fibre to transmit an infrared signal to a detector.

the optical materials chosen, sapphire $\left(\mathrm{Al}_{2} \mathrm{O}_{3}\right)$ and fused silica $\left(\mathrm{SiO}_{2}\right)$, are transparent to microwave radiation allowing for in situ, non-contact temperature measurements during heating and filter regeneration.

Precise thermal mapping of the filter is achieved by measuring the surface temperature at various locations within the filter. The goal of this research is to investigate the goniometric characteristics of optical fibres with various tip geometries. The results of this study identify areas along the filter wall from which the optical fibre tip accepts incident radiation.

Previous research on biomedical engineering applications has focused on the delivery of laser light through optical fibres with various tip geometries [3-11]. Optical fibre endoscopes with flat tip geometry are constrained to forward firing in tissue removal procedures unless the fibre tip is curved by specially designed distal end attachments. With the introduction of side-firing optical fibres for endoscopic operations in ophthalmology and urology, tissue ablation along organ walls is achieved without physically redirecting the tip of the fibre [4-7]. By altering the fibre tip geometry to a bevelled surface, the property of total internal reflection is exploited. Laser radiation travelling through the fibre reflects at the angled end surface, exiting at a large angle off the optical axis. As shown through experiment and ray tracing techniques, variation in the angle of the bevelled tip dictates the exit angle of the laser light [4].

Systematic research regarding the acceptance of radiation from an external source is not readily available and is therefore studied in this work. Three tip geometries are investigated in this study. The flat, $45^{\circ}$ bevelled and bent and polished tip geometries are shown in figures 2(a)-(c). In this study, the fibre with the bent and polished tip geometry is made of fused silica while the other two fibres are made of sapphire.

The numerical aperture (NA) of an optical fibre is an important characteristic in understanding the acceptance of incident radiation by the fibre tip. Nubling and Harrington [12] have studied the optical properties of single-crystal sapphire optical fibres. With a refractive index of 1.75, an unclad flat tip sapphire fibre in air has a theoretical NA $>1$ suggesting that the sapphire fibre will transmit all rays incident upon the input face regardless of the angle. In practice, this is not the case since bulk and surface defects originating from the manufacturing process scatter the light into modes that do not transmit through the length of the optical fibre. Using the angular scan method as dictated by the International Electrotechnical Commission (IEC) [13], the effective (versus theoretical) NA, $\mathrm{NA}_{e f f}$, of each fibre is therefore defined and investigated in this study.
It is well known that the flat tip optical fibre will accept radiation from a region directly forward. As for the bent and polished tip geometry, no previous research about the predicted viewing area has been found. By firing an argon laser through the $45^{\circ}$ angled tip fibre encapsulated within a cylinder of photographic paper, Russo et al $[6,7]$ showed that the output beam has a primary and secondary component. The primary component is located perpendicular to the optical axis exiting through a meridional surface while the secondary is roughly $15^{\circ}$ off the optical axis in the same direction. Though a visible interpretation of the output characteristics of a $45^{\circ}$ angled tip optical fibre was provided, definitive reasoning for the two regions observed was not investigated. The acceptance of incident radiation into an optical fibre is expected to exhibit a similar response as that of radiation being expelled. This study quantitatively compares and identifies the regions and intensities of radiation accepted by the optical fibres as a function of the various tip geometries.

The experimental set-up, equipment and alignment procedures are first introduced. Results from the experiments performed on the three fibres are subsequently presented and analysed. Discussion of the results and applications for temperature measurement are then presented.

\section{Experimental set-up}

Figure 3 shows the overall experimental set-up used to measure the effective NA of the different fibre tip geometries. The following sections discuss the angular scan method, radiation sources, infrared detectors and optical fibres used in this study and present the alignment procedures followed for the experiments.

\subsection{Angular scan method}

The experimental procedure employed to measure the NA of the optical fibres was the far-field pattern (FFP) method. This is a widely used method for measuring the acceptance angle of optical fibres [14-16]. The experiments conducted in this research were based on instructions defined by the IEC standard CEI/IEC 60793-1-43 [13]. The main deviation from the procedures dictated by the IEC standard is that, in this research, radiation is incident upon the fibre instead of being emitted from the fibre. This altered approach is intended to simulate the radiation acceptance capabilities of the optical fibre within the ceramic filter.

A rotary table, as illustrated in figure 3 , is used to rotate the radiation source around the tip of the fibre, which is aligned at the centre of the rotary table. The fibre is held fixed by a fibre clamp mounted to a post isolated from the rotary table. The proximal end of the optical fibre is connected directly to an infrared detector via an SMA 905 connector.

Two angles are used to define the rotation and orientation between the fibre and radiation source. The rotation angle, $\alpha$, defines the angular position of the radiation source relative to the central axis of the fibre. As shown in the top view in figure $3, \alpha=0^{\circ}$ is defined by the radiation source positioned along the extended axis of the optical fibre. The angle $\alpha$ is positive when the rotary table rotates counterclockwise off the $0^{\circ}$ angle and negative otherwise. 

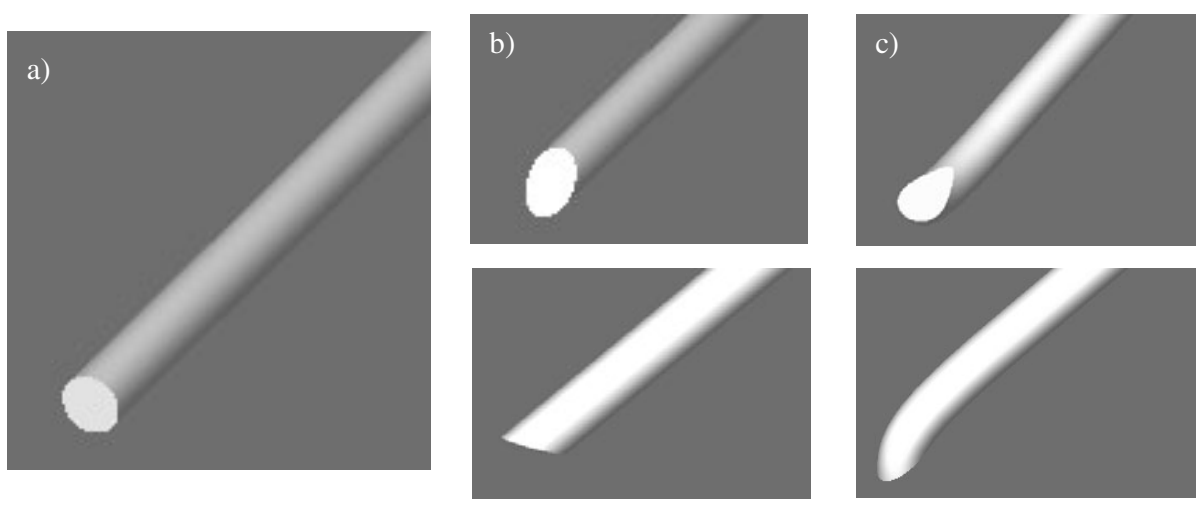

Figure 2. Illustration of the three tip geometries of the optical fibres examined for this study: (a) flat tip, (b) $45^{\circ}$ angled tip (two orientation views) and (c) bent and polished tip (two orientation views).

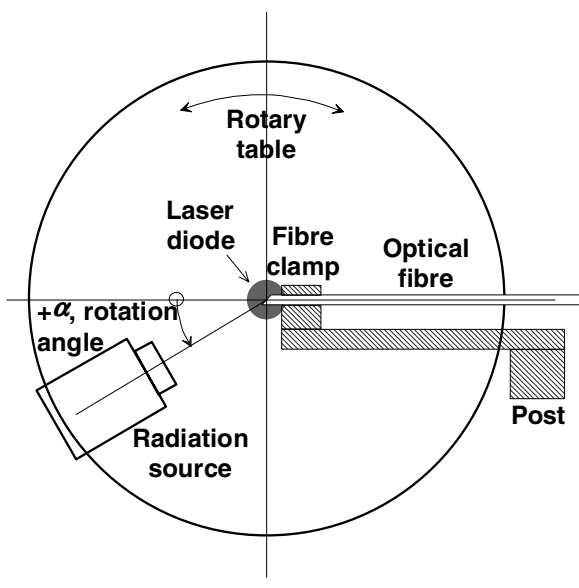

Top view

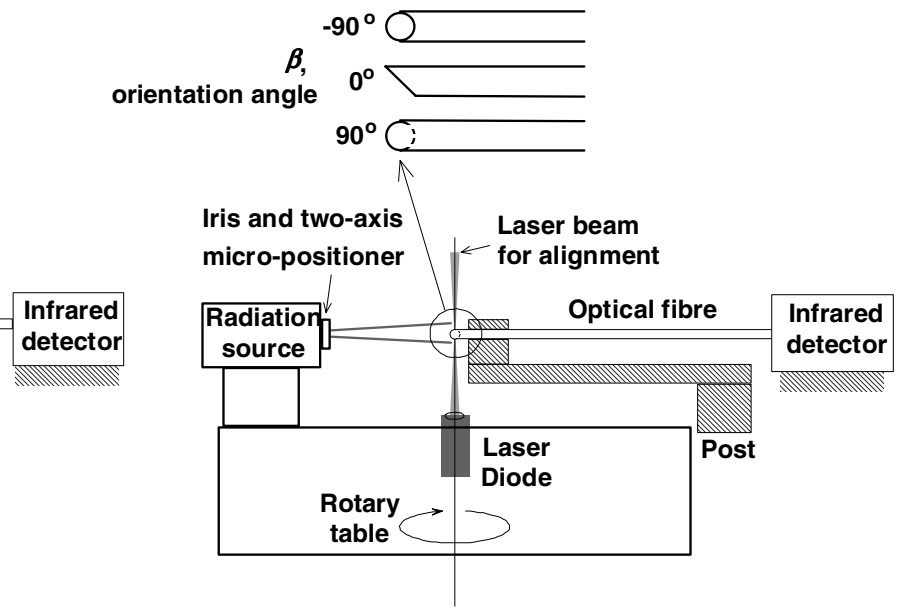

Front view

Figure 3. Experimental set-up and definition of rotation angle $(\alpha)$ and orientation angle $(\beta)$.

The orientation angle, $\beta$, is applied to characterize the angular position of the polished face of the angled and bent tip optical fibres. As shown in the fibre tip close-up in the front view of figure $3, \beta=0^{\circ}$ is defined by the cut face of the fibre tip pointed downward. The orientation shown in the top view of figure 3 is $\beta=90^{\circ}$. In this orientation, when the radiation source is located at $\alpha=90^{\circ}$, the incident photons encounter only the cylindrical surface of the optical fibre.

\subsection{Radiation sources, infrared detectors and optical fibres}

Two radiation sources for high and low-temperature measurements are used in this study. For high temperatures, the $3100 \mathrm{~K}$ tungsten halogen light source (Ocean Optics Model LS-1) was selected. The emitted radiation is channelled down a set of optical tubes designed to encapsulate the entire spatial output. At the termination of the tubes is a calibrated iris adjustable from 1 to $12 \mathrm{~mm}$, graduated in $0.5 \mathrm{~mm}$ increments. An Inconel cavity, prepared such that its characteristic emissivity approaches that of a blackbody [17], was used as a radiation source for low-temperature measurements. Affixed to the face of the radiation source, a plate with a centrally located $3 \mathrm{~mm}$ diameter hole provided an aperture for the lowtemperature measurements. The aperture further improves a uniform temperature distribution within the blackbody by reflecting a substantial portion of the radiation back into the cavity.

At high temperatures, a 2048-element linear silicon CCD array spectrometer (Ocean Optics Model USB2000) is utilized. This detector is configured to identify radiation within a wavelength range of 720-980 nm. At lower temperatures, a lead sulfide $(\mathrm{PbS})$ photoconductor, manufactured by electrooptical systems, with an operating wavelength in the range of $1-2.8 \mu \mathrm{m}$, peaking at $2.5 \mu \mathrm{m}$, is used. The sensor is cooled thermoelectrically to $243 \mathrm{~K}$ and the incoming dc signal is mechanically converted to an ac signal with a chopper set to $250 \mathrm{~Hz}$.

Two of the three fibres examined are unclad, multimode, single-crystal sapphire $\left(\mathrm{Al}_{2} \mathrm{O}_{3}\right)$ with a diameter of $0.425 \mathrm{~mm}$ and a length of $1 \mathrm{~m}$. The distal ends of the flat and angled tip fibres, as shown in figures 2(a) and (b), were polished at an angle relative to the optical axis of $90^{\circ}$ and $45^{\circ}$ respectively. The third optical fibre has a bent and polished tip geometry, as shown in figure 2(c). The polished tip is flush with the cylindrical wall of the fibre along the meridional surface intended for gathering emitted radiation from a direction perpendicular to the optical axis. This fibre, essentially a lightpipe, is made of fused silica with a diameter of $2 \mathrm{~mm}$ and a length of $0.3 \mathrm{~m}$. The proximal end of all three optical fibres 
has an SMA 905 connection allowing for efficient coupling of the transmitted radiation directly into the sensor. Optically, sapphire transmits wavelengths over a range from 0.45 to $3.5 \mu \mathrm{m}$ and fused silica transmits wavelengths over a range from 0.25 to $2 \mu \mathrm{m}$. The refractive index at a wavelength of $850 \mathrm{~nm}$ is 1.75 for sapphire and 1.45 for fused silica $[18,19]$.

The two sapphire optical fibres were flexible and experienced nominal bending along the metre length of the fibre. Within the range examined, no wavelength dependent bend losses were observed. Since the silica lightpipe was rigid, no bending along the length was possible.

\subsection{Procedures for alignment}

The precise alignment of various components utilized in this research was paramount in achieving accurate, reproducible results. The following sections detail the steps taken to align the laser diode, optical fibre and radiation source.

2.3.1. Aligning the fibre tip to the centre of the rotary table. As shown in figure 3, a laser diode is installed in the centre of the rotary table to assist in marking a reference point for placement of the fibre tip at the central axis. In front of the laser, a focusing lens was used to converge the coherent laser light to a small spot on a thin, translucent plastic target grid. This plastic sheet is held by an articulated post assembly mounted external to the rotary table and placed at the same height where the optical fibre tip will be located.

If the laser is not perfectly centred on the rotary table, the focused spot on the plastic sheet will displace as the rotary table is revolved through $180^{\circ}$. Placement of the laser diode is minutely adjusted over consecutive procedures so that the spot on the plastic sheet stays in essentially the same location through the entire $180^{\circ}$ rotation. The spot is therefore located very close to the central axis of the rotary table. The optical fibre is then moved into position through the use of a micropositioning linear translator such that its tip is centred at the middle of the focused spot.

2.3.2. Aligning the radiation source. Alignment of the radiation source involves two steps: (1) rough and (2) fine alignment. Through visual inspection, rough alignment involves positioning the radiation source such that the emitted light is aimed directly at the fibre tip. The incident radiation spot is then finely aligned by adjusting a two-axis linear micropositioner until a maximum intensity value is observed at the detector. At this position, the radiation source and the optical fibre are aligned along the optical axis.

2.3.3. Aligning the fibre orientation. Alignment of the angled tip fibre in the $0^{\circ}$ orientation $\left(\beta=0^{\circ}\right)$ was facilitated through the use of the laser diode. When the laser light is incident on the cut face precisely at $0^{\circ}$, a spot is reflected along the optical axis in front of the fibre. Using a white piece of plastic, the visible spot is an approximate guide to aligning the fibre into the correct orientation.

With the optical fibre in the $90^{\circ}$ orientation $\left(\beta=90^{\circ}\right)$ relative to the optical axis and the radiation source in the $90^{\circ}$ position $\left(\alpha=90^{\circ}\right)$, the greatest amount of radiation reflects off the cut face and travels down the fibre. Using the spectrometer to monitor the level of intensity, the highest value is approximated as the correct $90^{\circ}$ orientation.

Alignment of the fused silica fibre into the two orientations $\left(\beta=90^{\circ}\right.$ and $0^{\circ}$ ) was achieved through visual means since the diameter is larger and easier to position within an error level comparable to the angle tip fibre.

\section{Experimental results}

The relative intensity profiles of the three optical fibres examined are presented in following sections. The results obtained at low temperatures closely mimic those observed during high-temperature measurements due principally to the negligible impact the wavelength-dependent refractive index has on the effective NA within the wavelength range examined. Accordingly, only the high-temperature results are presented in this paper.

\subsection{Flat tip fibre}

Figure 4 shows the relative intensity profile of the flat tip sapphire optical fibre at high temperature with the aperture diameter set to $1 \mathrm{~mm}$. Each experimental run was conducted at increments of $0.5^{\circ}$ over a range of $\pm 14^{\circ}$. The two profiles essentially overlap, validating the repeatability of the experimental set-up. Angular scan experiments were also conducted using a $3 \mathrm{~mm}$ aperture diameter at high temperature. All tests resulted in relative intensity curves similar to that shown in figure 4 [20].

The IEC standard [13] defines the fibre acceptance angle as the angle where $5 \%$ of the peak relative intensity exists. Consequently, the effective NA, $\mathrm{NA}_{e f f}$, is computed by taking the sine of that $5 \%$ angle. Based on the standard and the results from figure 4 , the flat tip sapphire fibre examined in this study has an effective acceptance angle, $\theta_{\text {eff }}$, of $9^{\circ}$ corresponding to an effective NA of 0.156 . This is much smaller than the theoretical $90^{\circ}$ acceptance angle. Similar observations for unclad sapphire fibres have been reported in previous research [12]. This is probably a consequence of signal attenuation experienced by higher modes as a function of fibre length. Since the length of the optical fibre examined was $1 \mathrm{~m}$, over 2000 diameters, it is unlikely that further attenuation will be experienced as the fibre length is increased. Conversely, it is expected that as the fibre length is decreased, $\mathrm{NA}_{\text {eff }}$ will increase toward unity. However, this was not verified by investigation. In section 4 , discussion of this observation leads to a better understanding of the relative intensity profile of the angled tip fibre.

\section{2. $45^{\circ}$ angled tip fibre}

Figure 5 shows the relative intensity profiles of the $45^{\circ}$ angled tip sapphire fibre at $0^{\circ}$ and $90^{\circ}$ orientation angles $(\beta)$. At $\beta=90^{\circ}$, the relative intensity peaks at a rotation angle $(\alpha)$ of $93.6^{\circ}$. A small hump, reaching to about $8.7 \%$ of the maximum relative intensity, can be seen at roughly $18^{\circ}$. A local minimum, or valley, between the peak and hump is located at $\alpha=43^{\circ}$. At $\beta=0^{\circ}$ and $\alpha=0^{\circ}$, the relative intensity compared with the peak at $\beta=90^{\circ}$ and $\alpha=93.6^{\circ}$ is very low, only about $6.5 \%$. This indicates that most radiation received by the 


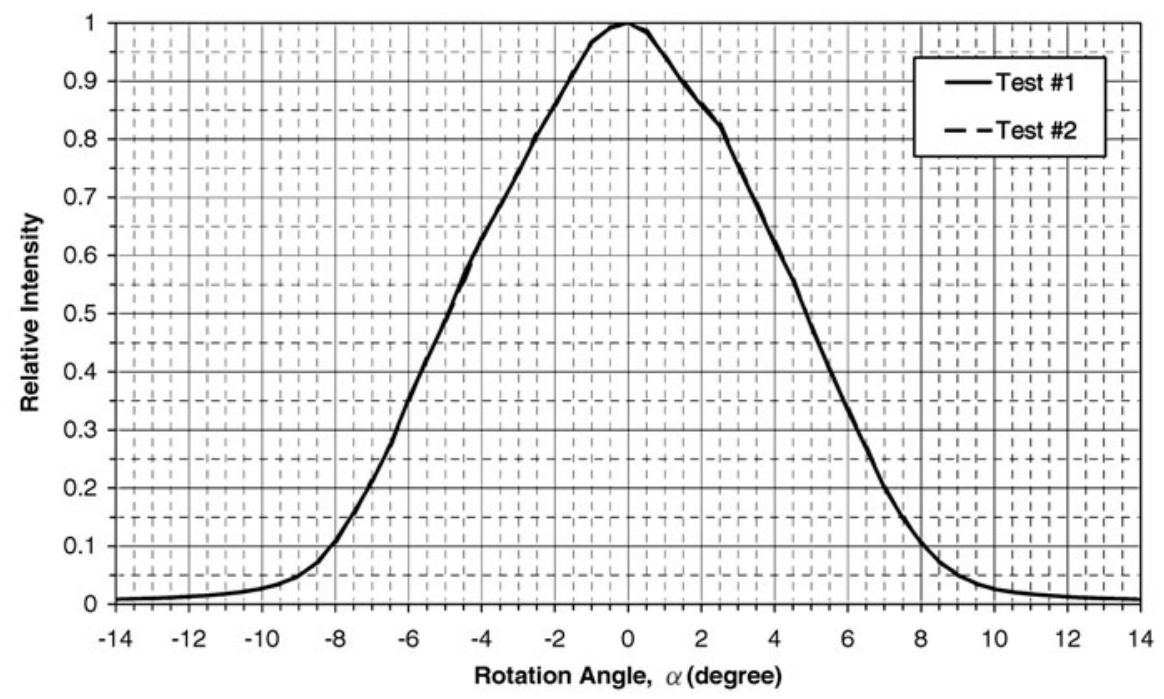

Figure 4. Relative intensity profile for the flat tip sapphire fibre at high temperature with a $1 \mathrm{~mm}$ diameter iris. The results from tests 1 and 2 are practically identical. The graphs are therefore overlaid.

angled tip optical fibre is in the $\beta=90^{\circ}$ plane, particularly concentrated in the direction perpendicular to axis of the optical fibre. Using the IEC 5\% relative intensity guideline, the effective acceptance region is between $0^{\circ}<\alpha<30^{\circ}$ and $65^{\circ}<\alpha<125^{\circ}$ for $\beta=90^{\circ}$ and between $-15^{\circ}<\alpha<15^{\circ}$ for $\beta=0^{\circ}$.

The two angular scans at $\beta=0^{\circ}$ and $90^{\circ}$ cross each other at $\alpha=0^{\circ}$. The same $6.5 \%$ relative intensity at $\alpha=0^{\circ}$ further validates the accuracy and repeatability of the experimental set-up.

\subsection{Fibre with bent and polished tip}

Figure 6 shows the two angular scan results for the bent and polished tip fibre at $\beta=0^{\circ}$ and $90^{\circ}$ superimposed on the same graph. The relative peak is located at $\alpha=80^{\circ}$, close to the peak location observed for the angled tip fibre. However, this fibre is capable of receiving radiation across a wider range of $\alpha$. Based on the IEC standard of $5 \%$ relative intensity, the effective acceptance angle covers the broad region between $-90^{\circ}<\alpha<117^{\circ}$ for $\beta=90^{\circ}$ and $-30^{\circ}<\alpha<30^{\circ}$ for $\beta=0^{\circ}$.

In the $\beta=90^{\circ}$ plane, a distinct peak occurs at $\alpha=-45^{\circ}$ with a value of $86 \%$ of the relative maximum intensity. The possible cause for this peak is discussed in the next section. Furthermore, at $\alpha=0^{\circ}$ the two angular scans meet at the same $16 \%$ relative intensity, once again validating the accuracy and repeatability of the experimental configuration.

\section{Discussion}

This section attempts to explain the intensity profile results obtained from the angular scan experiments by applying Snell's law and the Fresnel equations to ray tracing techniques. Additional discussion is directed at optical fibre applications in diesel after-treatment filter temperature measurement.

\subsection{Flat tip fibre}

The measured $9^{\circ}$ effective acceptance angle is significantly smaller than the theoretical $90^{\circ}$ acceptance angle of the unclad, multi-mode flat tip sapphire fibre. According to Nubling and Harrington [12], this is caused by surface and bulk defects which scatter light at the surface interface and along the length of the fibre. Similar observations of small effective acceptance angles, around $10^{\circ}$, for flat tip sapphire fibres have been reported by Kottmann and Stenzel [21]. Figure 7(a) shows ray tracing of light incident upon the flat tip fibre surface at the $9^{\circ}$ effective acceptance angle. On the external surface of the fibre tip, using the Fresnel equations and refractive indices of 1.75 and 1.00 for sapphire and air [22], 92.7 and $7.3 \%$ respectively of the radiation is transmitted and reflected. From the Fresnel equations, calculated values of transmittance and reflectance as a function of the incident angle at the air/sapphire interface are plotted in figure 8.

As shown in figure 7(a), at the maximum incident angle of $9^{\circ}$ the internal incident angle is $84.9^{\circ}$. This can be considered an effective critical angle for total internal reflection within the fibre. This is significantly larger than the $34.9^{\circ}$ theoretical critical angle calculated based on the Snell's law. This result indicates that a large internal incident angle is necessary to transmit the entire light signal from the fibre tip to the detector at the distal end of the fibre. This observation is applied to understand the results for $45^{\circ}$ angled tip and bent tip fibres.

\section{2. $45^{\circ}$ angled tip fibre}

As shown in figure 5, the peak, hump and valley can be identified from the $45^{\circ}$ angled tip fibre response in the $\beta=$ $90^{\circ}$ orientation. To help explain these three characteristics, table 1 lists the variation in external incident angle, external transmittance and reflectance, and internal incident angle. Results from table 1 and the ray tracing techniques help quantitatively explain the features of the $45^{\circ}$ angled tip fibre angular scans. 

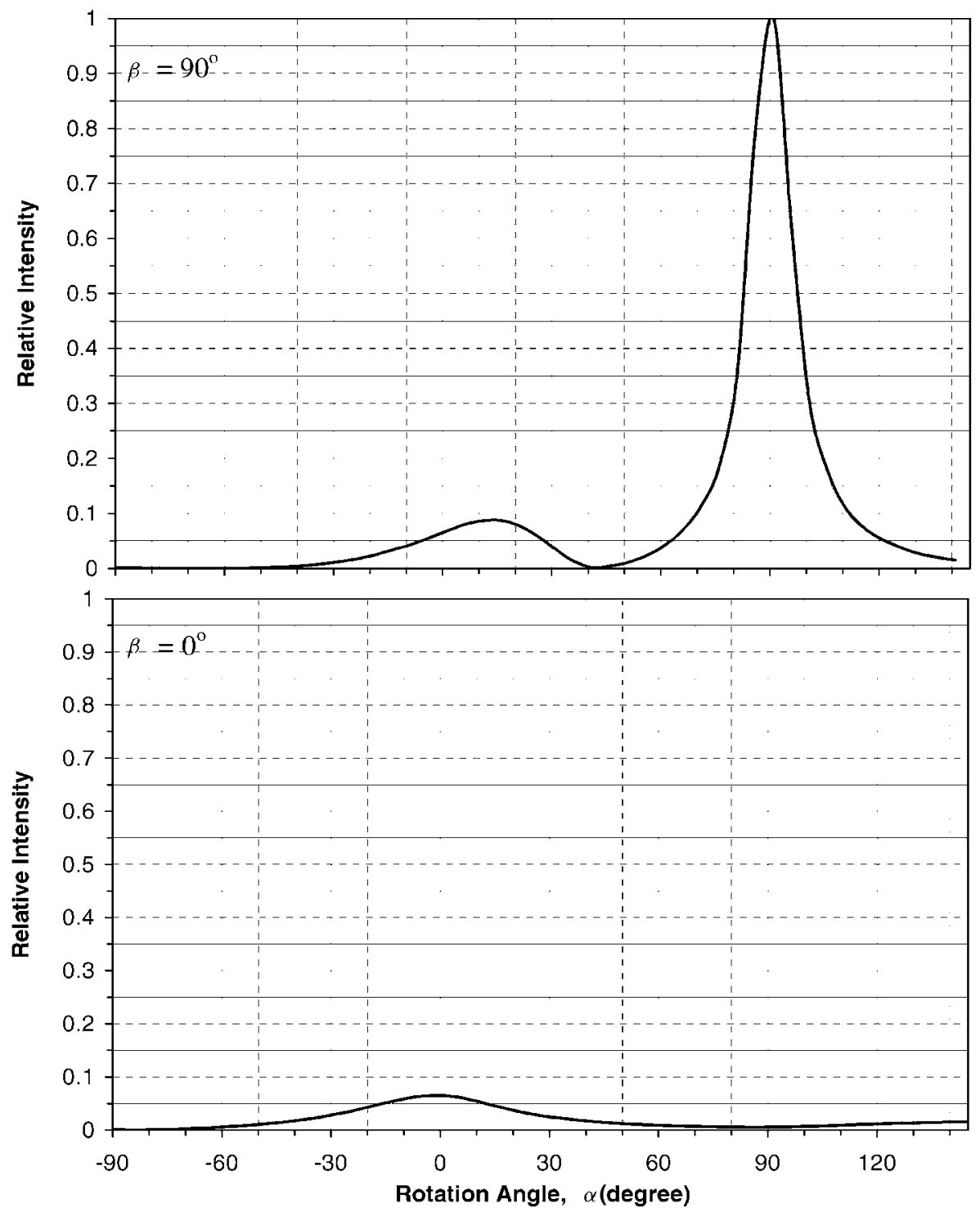

Figure 5. Relative intensity profile for the $45^{\circ}$ angled tip sapphire fibre at high temperature in the $\beta=90^{\circ}$ (top) and $0^{\circ}$ (bottom) orientation.

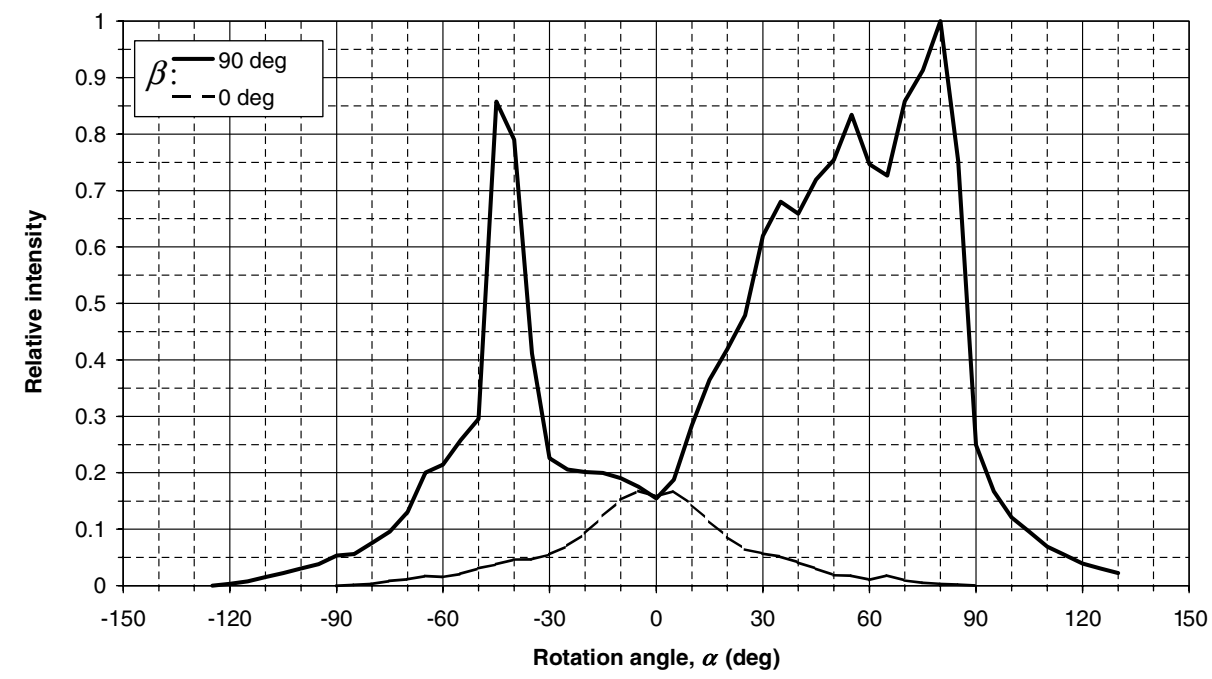

Figure 6. Relative intensity profile for the fibre with bent and polished tip at high temperature. 


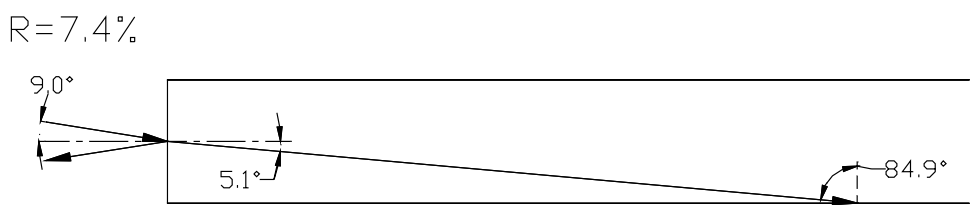

a) Flat tip fibre with $9^{\circ}$ incident angle.

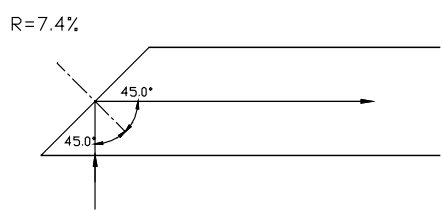

b) $\alpha=90^{\circ}$ for $45^{\circ}$ angled tip fibre.

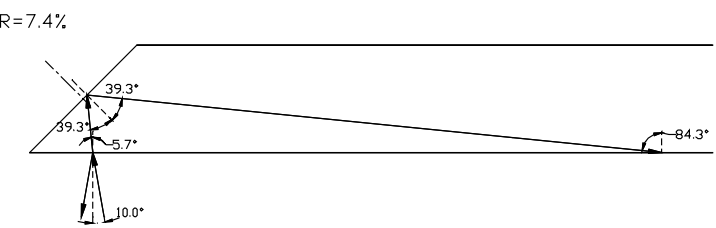

c) $\alpha=100^{\circ}$ for $45^{\circ}$ angled tip fibre.

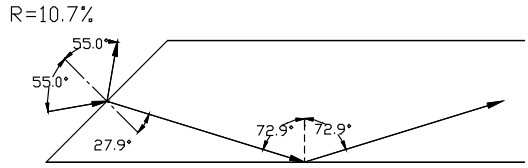

d) $\alpha=10^{\circ}$ for $45^{\circ}$ angled tip fibre.
$\mathrm{R}=12.8 \%$

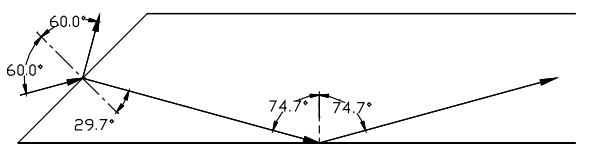

e) $\alpha=15^{\circ}$ for $45^{\circ}$ angled tip fibre.

$\mathrm{R}=15.9 \%$

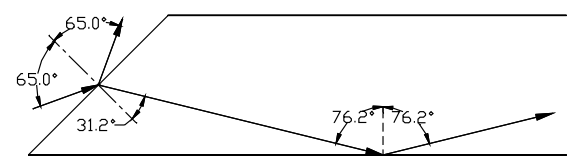

$\mathrm{R}=100 \%$

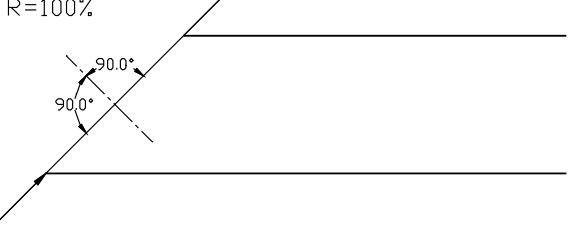

g) $\alpha=45^{\circ}$ for $45^{\circ}$ angled tip fibre.

Figure 7. Ray tracing in the cross-sectional plane of the flat tip optical fibre and the $45^{\circ}$ angled tip optical fibre at $\beta=90^{\circ} ; R$ : the percentage reflectance at the air/sapphire interface.

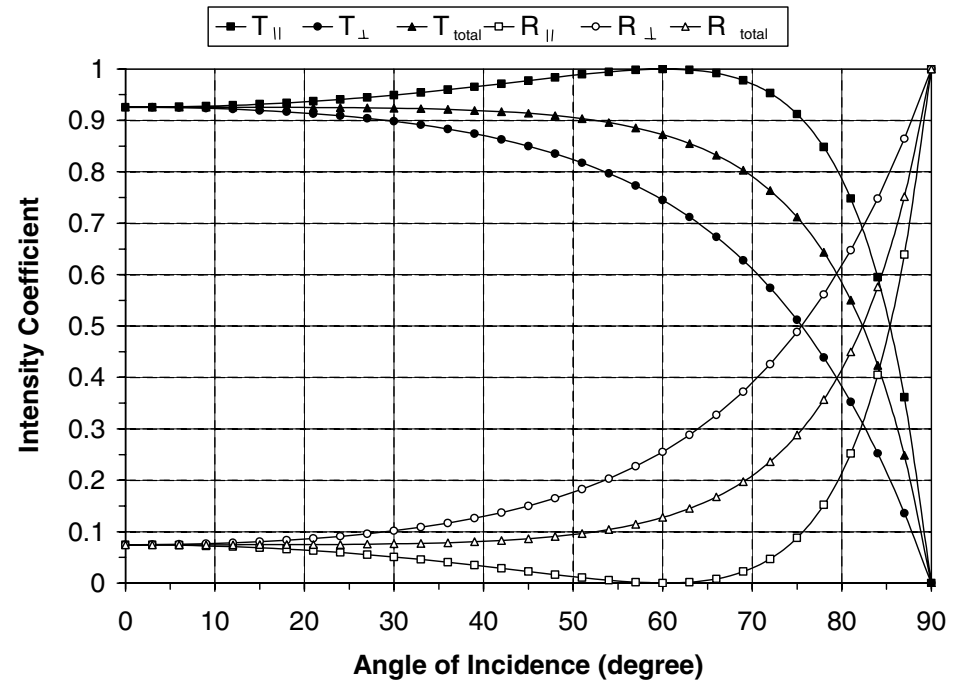

Figure 8. Perpendicular and parallel components and total transmittance and reflection for the air/sapphire interface.

The path of a single ray at $\alpha=90^{\circ}$ and $100^{\circ}$ in the $\beta=90^{\circ}$ plane is shown in figures 7(b) and (c) respectively. A high percentage of the incident light is transmitted through the fibre in these positions. A $90^{\circ}$ turning prism effect, a consequence of total internal reflection with the surface of the $45^{\circ}$ angled tip, directs the light at large internal incident angles to the detector accounting for the peak near $\alpha=90^{\circ}$. This result implies that lower modes produce the greatest response for this sapphire optical fibre.

Experimental results in figure 5 show the location of the peak at $\alpha=93.6^{\circ}$, slightly different from the theoretical value of $\alpha=90^{\circ}$ shown in figure 7(b). This discrepancy can be 
Table 1. Internal incident angle and percentage of reflectance and transmittance as a function of external incident angle for the $45^{\circ}$ angled tip fibre.

\begin{tabular}{ccccc}
\hline $\begin{array}{l}\text { Angular position of } \\
\text { radiation source, } \alpha \\
(\text { deg })\end{array}$ & $\begin{array}{l}\text { External } \\
\text { incident angle } \\
(\mathrm{deg})\end{array}$ & $\begin{array}{l}\text { Total external } \\
\text { reflectance } \\
(\%)\end{array}$ & $\begin{array}{l}\text { Total external } \\
\text { transmittance } \\
(\%)\end{array}$ & $\begin{array}{l}\text { Internal } \\
\text { incident angle } \\
(\mathrm{deg})\end{array}$ \\
\hline-90 & 45 & 8.6 & 91.4 & 21.2 \\
-45 & 0 & 7.4 & 92.6 & 45.0 \\
0 & 45 & 8.6 & 91.4 & 68.8 \\
10 & 55 & 10.7 & 89.3 & 72.9 \\
15 & 60 & 12.8 & 87.2 & 74.7 \\
20 & 65 & 15.9 & 84.1 & 76.2 \\
30 & 75 & 28.8 & 71.2 & 78.5 \\
45 & 90 & 100.0 & 0.0 & 79.8 \\
60 & 30 & 7.6 & 92.4 & 73.4 \\
75 & 15 & 7.4 & 92.6 & 81.5 \\
90 & 0 & 7.4 & 92.6 & 90.0 \\
100 & 10 & 7.4 & 92.6 & 84.3 \\
120 & 30 & 7.6 & 92.4 & 73.4 \\
\hline
\end{tabular}

contributed to two sources. One is nominal misalignment of the angular scan experimental set-up, particularly in setting the orientation angle $\beta$. Another possible source of error is the potential deviation from manufacturing a true $45^{\circ}$ cut angle at the fibre tip.

Figure 5 also shows the hump at $\alpha=18^{\circ}$. From ray tracing techniques, the external reflectance and transmittance at $\alpha=10^{\circ}, 15^{\circ}$ and $20^{\circ}$ as well as the internal reflection are shown in figures 7(d)-(f). In the region of the hump $\left(10^{\circ}<\right.$ $\alpha<20^{\circ}$ ), the internal incident angle is increasing, which, as demonstrated in the analysis of the flat tip fibre, is critical to efficiently direct light toward the detector. However, as the rotation angle $\alpha$ increases from $10^{\circ}$ to $20^{\circ}$, the corresponding transmittance at the $45^{\circ}$ angled tip surface decreases, as shown in figures 7(d)-(f), figure 8 and table 1. These two competing factors may be responsible for the hump observed at $\alpha=$ $18^{\circ}$. Another possible explanation of the hump is the $100 \%$ transmission of the parallel component of the incident radiation at $60^{\circ}$ corresponding closely with Brewster's angle [22], as shown in figure 8 . By offsetting the results $3^{\circ}$ due to potential misalignment of $\beta$, the $\alpha=15^{\circ}$ position, corresponding to a $60^{\circ}$ incident angle with the angled tip surface normal, matches the peak for maximum transmission of the parallel component of the light incident on the surface. This hypothesis requires further in-depth study for verification.

The valley at $\alpha$ approaching $45^{\circ}$ can be analysed using the ray tracing method. As shown in figure $7(\mathrm{~g})$, the incident ray is parallel to the cut surface with $0 \%$ transmission, thereby causing the valley.

It is important to note that all $\beta=90^{\circ}$ ray tracings represent a single two-dimensional cross-sectional plane of the cylindrical fibre. In reality, the radiation is incident on a cylindrical curved surface, resulting in a much more complicated system of reflected and transmitted rays.

\subsection{Bent tip fibre}

Discussion of the angled tip fibre can help explain the response observed from the bent tip fibre experiments shown in figure 6 . In the $\beta=90^{\circ}$ orientation, a major peak is observed at $\alpha=80^{\circ}$. This is likely to be due to light transmitting through the flat polished tip reflecting off the internal curved surface
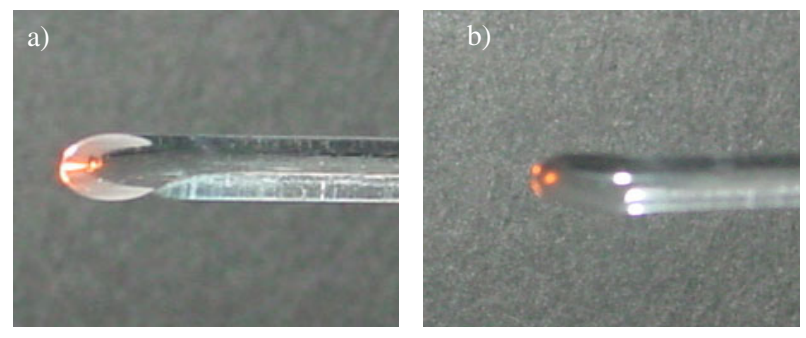

Figure 9. Light projected from a bent and cut tip fibre for (a) a polished flat surface and (b) a curved surface opposite the polished flat surface.

(This figure is in colour only in the electronic version)

at an internal incident angle resulting in the least number of modes within the fibre. This is similar to what is experienced by the angled tip fibre in the location around the $\alpha=90^{\circ}$ peak. The zigzag pattern observed for $\alpha$ between $30^{\circ}$ and $70^{\circ}$ is probably caused by complicated three-dimensional reflection and transmission from imperfections along the bent exterior and interior surfaces. At $\alpha=-45^{\circ}$, another major peak, registering $86 \%$ relative intensity, is experienced. A possible explanation for this result is that the transmitted radiation is reflecting off the internal planar polished surface at an angle similar to the low mode response observed at $\alpha=80^{\circ}$. As for the $\beta=0^{\circ}$ orientation, the expected symmetric response about the optical axis is evident.

A qualitative representation of the intensity profile is presented in figures 9(a) and (b). A $3100 \mathrm{~K}$ tungsten halogen light source is coupled directly to the proximal end of the fibre such that the radiation output can be visually observed at the bent fibre tip. From figure 9(a), the characteristic broad intensity range from $\alpha=80^{\circ}$ through roughly $30^{\circ}$ at the bent fibre tip is apparent. Figure 9(b) provides a distinct view of the second major peak observed at $\alpha=-45^{\circ}$ as well as the existence of two smaller irradiance spots along the very edge of the fibre tip. The gap between the two spots is coincident with the optical axis of the fibre and is probably represented by the central dip observed in the $\beta=0^{\circ}$ orientation intensity profile. 


\subsection{Signal intensity}

Comparing the flat, $45^{\circ}$ angled tip and bent tip fibres, there is a significant difference in the signal intensity received by the detector. The spectrometer integration time (IT) provides an indication of the radiation-gathering capability of the optical fibres. For the flat tip fibre, the IT was set to $27 \mathrm{~ms}$ such that the maximum spectral output was roughly 3500 counts. Each count, according to the spectrometer's manufacturer, corresponds to receiving about 86 photons. In order to register the same 3500 counts level for the $45^{\circ}$ angled tip and the bent tip fibres, the IT was dramatically increased to $1200 \mathrm{~ms}$.

The flat and $45^{\circ}$ angled tip fibres have the same diameter, length and material. The difference in IT settings indicates that the peak in the flat tip fibre receives 44 times more photons than the peak in the $45^{\circ}$ angled tip fibre. An experiment was conducted in which the tungsten halogen light source was coupled to the proximal end of the fibre such that the intensity of the light emitted from the fibre tip could be observed. Visually, there is a markedly higher intensity of light emitted from the flat tip fibre when compared with the angled tip fibre.

\subsection{Application to diesel exhaust filter temperature measurement}

The ideal optical fibre tip geometry for temperature measurement applications in diesel exhaust after-treatment will have only one peak at $\alpha=90^{\circ}$, perpendicular to the fibre axis. This physically translates to an optical fibre looking at a precise location along the wall of the filter. Among the three fibre tips examined in this study, the $45^{\circ}$ angled tip fibre is the closest to meeting such a requirement. As shown in figure 1 , with the $45^{\circ}$ angled tip fibre inside the filter cavity, a small distance is maintained between the fibre tip and the filter wall. Due to gravity, the fibre will bend slightly. The high elastic modulus of the sapphire is helpful in reducing the deflection of the fibre due to weight-induced bending. Using the orientation of angled tip shown in figure 1, the majority of the radiation emission received by the detector is from the area A underneath the fibre tip. The light emission from area B in front of the fibre tip makes some, relatively insignificant, contribution to the intensity of the signal received by the detector. Areas A and $\mathrm{B}$ correspond to the intensity peak and hump in figure 4 at $\alpha=90^{\circ}$ and $15^{\circ}$, respectively.

\section{Concluding remarks}

The spatial intensity profiles of three optical fibres with flat, $45^{\circ}$ angled and bent and polished tip geometries were investigated for their potential application in non-contact pyrometric temperature measurement of diesel exhaust aftertreatment filters. The measured effective acceptance angle of the flat tip sapphire fibre was much smaller than the theoretical acceptance angle and matched that reported by other researchers. The $45^{\circ}$ angled tip fibre exhibited a peak intensity perpendicular to the optical axis and a smaller hump intensity at a small angle off the optical axis in front of the fibre tip. The bent tip fibre showed an interesting two-peak pattern and a wide angular range of radiation acceptance.

This research concludes that the $45^{\circ}$ angled tip fibre, with a correct orientation of the tip surface, can be used to accurately detect thermal radiation emitted from a small area near the tip of the optical fibre. These characteristics make the $45^{\circ}$ angled tip sapphire optical fibre suitable for studying the spatial and temporal variation of temperature in diesel exhaust aftertreatment filters.

\section{Acknowledgments}

A portion of this research was sponsored by the Heavy Vehicle Propulsion Systems Materials Program, Office of Transportation Technologies, US Department of Energy. The authors further acknowledge the assistance provided by Dr Matthew Henrichsen of Cummins Inc. for providing the fused silica fibre and Dr Richard D Gould for many informative discussions.

\section{References}

[1] Mori K 1997 Worldwide trends in heavy-duty diesel engine exhaust emission legislation and compliance technologies Diesel Engine Combustion Processes Emission Control Technol. 1246 19-29 SAE Special Publications

[2] Koltsakis G C and Stamatelos A M 1997 Modes of catalytic regeneration in diesel particulate filters Ind. Eng. Chem. Res. 36 4155-65

[3] Denisov N A and Kravchenko I V 1996 Shape-dimension optimization of contact medical laser delivery systems Proc. SPIE 2928 46-57

[4] Mrochen M P R, Donitzky C and Seiler T 2001 Erbium: yttrium-aluminum-garnet laser induced vapor bubbles as a function of the quartz fiber tip geometry J. Biomed. Opt. 6 344-50

[5] Rol P, Utzinger U, Beck D and Niederer P 1994 Fiber beam shaping and ophthalmic applications Proc. SPIE 2330 56-62

[6] Russo V, Righini G, Sottini S and Trigari S 1985 Optical fibres for medical applications: output beam shaping Proc. SPIE 522 166-73

[7] Russo V, Righini G and Trigari S 1984 Side radiation optical fibres for medical applications Porphyrins in Tumor Phototherapy ed A Andreoni and R Cubeddu (New York: Plenum) pp 309-19

[8] Utzinger U and Richards-Kortum R R 2003 Fiber optic probes for biomedical optical spectroscopy J. Biomed. Opt. 8 $121-47$

[9] van Vleit R J, Molenaar D G, van Swol C F P, Boon T A and Verdaasdonk R M 1994 Optical characteristics of side firing fibers for laser prostatectomy Proc. SPIE 2328 102-9

[10] Verdaasdonk R M and van Swol C F P 1995 What makes a fiber tip do the job: an optical and thermal evaluation study Proc. SPIE 2396 37-45

[11] Verdaasdonk R M and Borst C 1990 Modified fiber tips: optical and thermal characteristics Proc. SPIE 1649 172-83

[12] Nubling R K and Harrington J A 1997 Optical properties of single-crystal sapphire fibers Appl. Opt. 36 5934-40

[13] International Standard 2001 CEI IEC 60793-1-43 Part 1-43: measurements methods and test procedures-numerical aperture International Electrotechnical Commission (Geneva, 2001)

[14] Murata H 1996 Handbook of Optical Fibers and Cables 2nd edn (New York: Dekker)

[15] Kuecken J A 1980 Fiberoptics (Pennsylvania: TAB Books)

[16] Kao C K 1988 Optical Fibre (London: P Peregrinus)

[17] Richmond J C, Dewitt D P and Hayes W D Jr 1964 Procedures for precise determination of thermal radiation properties National Bureau of Standards Technical Note 252 (Washington, DC: US Govt Printing Office)

[18] Tropf W J and Thomas M E 1998 Aluminum oxide $\left(\mathrm{Al}_{2} \mathrm{O}_{3}\right)$ revisited Handbook of Optical Constants of Solids vol 3, ed E D Palik pp 653-77 
[19] Philipp H R 1985 Silicon dioxide $\left(\mathrm{SiO}_{2}\right)$ (glass) Handbook of Optical Constants of Solids ed E D Palik pp 749-61

[20] Boothe B J 2002 Goniometric characteristics of sapphire optical fibers MS Thesis North Carolina State University
[21] Kottmann J P and Stenzel C 1999 Characterization of flexible sapphire fibers in high-temperature pyrometers Sensors Materials 11-14 233-46

[22] Hecht E 2002 Optics 4th edn (San Francisco, CA Addison-Wesley) 